\title{
Experimental Investigation of Wave Velocity-Permeability Model for Granite Subjected to Different Temperature Processing
}

\author{
Guanghui Jiang, ${ }^{1}$ Jianping Zuo, ${ }^{1,2}$ Teng Ma, ${ }^{1}$ and Xu Wei ${ }^{1}$ \\ ${ }^{1}$ School of Mechanics and Civil Engineering, China University of Mining and Technology, Beijing 100083, China \\ ${ }^{2}$ State Key Laboratory of Coal Resources and Safe Mining, China University of Mining and Technology, Beijing 100083, China \\ Correspondence should be addressed to Jianping Zuo; zjp@cumtb.edu.cn
}

Received 12 July 2017; Revised 4 September 2017; Accepted 26 September 2017; Published 30 October 2017

Academic Editor: Paolo Fulignati

Copyright (C) 2017 Guanghui Jiang et al. This is an open access article distributed under the Creative Commons Attribution License, which permits unrestricted use, distribution, and reproduction in any medium, provided the original work is properly cited.

Understanding the change of permeability of rocks before and after heating is of great significance for exploitation of hydrocarbon resources and disposal of nuclear waste. The rock permeability under high temperature cannot be measured with most of the existing methods. In this paper, quality, wave velocity, and permeability of granite specimen from Maluanshan tunnel are measured after high temperature processing. Quality and wave velocity of granite decrease and permeability of granite increases with increasing temperature. Using porosity as the medium, a new wave velocity-permeability model is established with modified wave velocityporosity formula and Kozeny-Carman formula. Under some given wave velocities and corresponding permeabilities through experiment, the permeabilities at different temperatures and wave velocities can be obtained. By comparing the experimental and the theoretical results, the proposed formulas are verified. In addition, a sensitivity analysis is performed to examine the effect of particle size, wave velocities in rock matrix, and pore fluid on permeability: permeability increases with increasing particle size, wave velocities in rock matrix, and pore fluid; the higher the rock wave velocity, the lower the effect of wave velocities in rock matrix and pore fluid on permeability.

\section{Introduction}

Permeability is a physical parameter to measure the ability of a porous material to allow fluids to pass through it. There are various methods to measure the permeability, such as steadystate method, transient pulse method [1], and oscillating pore pressure method [2]. The existing methods require strict conditions and the measurement is time consuming. Thus, for exploitation of hydrocarbon resources and disposal of nuclear waste, it is of great importance to simplify the permeability measurement under extreme conditions (high temperature and high pressure).

Acoustic testing technique is one of the most effective tools to study the earth interior. Through acoustic testing technique, some characteristics can be obtained in rock, such as porosity, destiny, saturation, fluid type, pressure, temperature, and geometrical characteristics of cracks [3]. Based on experiments, some researchers found that ultrasonic velocity is more sensitive to porosity. Nara and Kaneko [4] used the P wave velocity to analyze the propagation of cracks in granite under different temperatures and humidity. They found that the propagation of cracks caused by stress corrosion can be monitored by $\mathrm{P}$ wave velocity well. Wave velocity is more sensitive to the change of rock mesostructure. Kuila et al. [5] investigated the ultrasonic velocity response of well-characterised low porosity shales from the Officer Basin in Western Australia to both isotropic and anisotropic stress fields and evaluated the velocity response to the changing stress field. The results indicate the magnitude and orientation of the stress anisotropy with respect to the shale microfabric have a significant impact on the velocity response to changing stress fields. Qiao and Zhao [6] obtained accurate $\mathrm{P}$ wave and $\mathrm{S}$ wave velocity data at pressures up to $180 \mathrm{MPa}$ on different core samples of sandstone in dry, water, and oil-saturated conditions from the Yanchang oilfield. The differences among the wave velocities 
in various conditions are mainly caused by the different rock microstructures.

Some researchers also developed empirical wave velocityporosity formulas. Wyllie et al. [7-9] found the monotone function between wave velocity and porosity. Raymer et al. [10] improved Wyllie's formula and proposed wave velocityporosity formula is an expression of divided function. The relationship between wave velocity and porosity in low porosity rocks is obviously different than the one in high porosity rocks.

Carman [11] proposed a formula to estimate permeability of porous media with some general parameters, such as porosity, superficial area, and particle size. Based on Carman's research, Coussy [12] proposed a more common form of Kozeny-Carman formula. Above research results indicate that permeability of rock is connected with porosity.

Rock permeability under high temperature usually needs to be measured for the engineering purposes such as coal gasification and nuclear waste disposal. With increasing temperature, permeability increases owing to the increase in number and size of pores and cracks. Scholars have researched the effects of temperature on rock properties from different aspects, such as breakage mesomechanism [13-16], mechanical property [17-20], acoustic property [2123], and permeability [22-25]. Few experimental equipments can measure the permeability directly at high temperature (above $150^{\circ} \mathrm{C}$ ). But the wave velocity measurement at high temperature can be obtained easily now. If the relationship between wave velocity and permeability can be established, the permeability of rock at high temperature can be obtained from wave velocity measurement.

Benson et al. [26, 27] modeled the permeability evolution of microcracked rocks with different void space geometries at elevated pressure and tried to establish the relationship of wave velocity and permeability. With reference to Benson's work, porosity is used as medium to establish a wave velocity-permeability model in this paper. Wave velocity and permeability of rock will be changed by high temperature processing to prove the new model. The ultimate goal is to measure the rock permeability indirectly by acoustic testing technique to provide practical guidance for exploitation of hydrocarbon resources and disposal of nuclear waste.

\section{Wave Velocity-Permeability Model of Granite}

An extended form of Kozeny-Carman formula considering matrix grains are spherical is shown as follows:

$$
k=\frac{d^{2}}{36 c} \frac{\varphi^{3}}{(1-\varphi)^{2}},
$$

where $c$ is Kozeny-Carman constant changed with grain shape [28]; $d$ is particle size; $\varphi$ is porosity.

Raymer et al. [10] improved Wyllie's formula:

$$
v=(1-\varphi)^{2} v_{m}+\varphi v_{\mathrm{fl}}, \quad \varphi<37 \%
$$

$$
\frac{1}{\rho v^{2}}=\frac{\varphi}{\rho_{\mathrm{fl}} v_{\mathrm{fl}}^{2}}+\frac{1-\varphi}{\rho_{m} v_{m}^{2}}, \quad \varphi>37 \%,
$$

where $\varphi$ is the rock porosity; $v_{r}, v_{\mathrm{fl}}$, and $v_{m}$ are $\mathrm{P}$ wave velocities in the rock, pore fluid, and rock matrix, respectively; $\rho, \rho_{\mathrm{fl}}$, and $\rho_{m}$ are densities of the rock, pore fluid, and rock matrix, respectively.

In this paper granite is with low porosity; only the first part of (2) is needed to calculate the porosity. Furtherly, the porosity measured by liquid saturation method at normal temperature $T_{0}$ is used as initial porosity $\varphi_{0}$ to improve (2).

Equation (2) is used to calculate porosity difference $\Delta \varphi$ between $\varphi$ at temperature $T$ and $\varphi_{0}$ at normal temperature $T_{0}$.

$$
\varphi=\varphi_{0}+\Delta \varphi
$$

According to (2),

$$
\Delta \varphi=\frac{-m-\sqrt{m^{2}+4 v_{m} \Delta v}}{2 v_{m}},
$$

where $m=-\sqrt{v_{\mathrm{fl}}^{2}+4 v_{m}\left(v_{0}-v_{\mathrm{fl}}\right)} ; \Delta v=v-v_{0} ; v_{0}$ is the wave velocity at normal temperature $T_{0}, \mathrm{~m} / \mathrm{s}$.

Combining (3) and (4), the porosity yields

$$
\varphi=\varphi_{0}-\frac{m+\sqrt{m^{2}+4 v_{m}\left(v-v_{0}\right)}}{2 v_{m}} .
$$

In heating process, the main change in rock is the change of pore and crack structures resulting in the changes in permeability and wave velocity. Therefore, there is clear correlation between permeability and wave velocity through the medium of porosity. Combining (1) and (5), the wave velocity-permeability model can be established.

Under given initial porosity $\varphi_{0}$ and initial wave velocity $v_{0}$, the relationship between permeability and wave velocity at any temperature yields

$$
k=\alpha \frac{\left(\varphi_{0}-\left(m+\sqrt{m^{2}+4 v_{m}\left(v-v_{0}\right)}\right) / 2 v_{m}\right)^{3}}{\left(1-\varphi_{0}+\left(m+\sqrt{m^{2}+4 v_{m}\left(v-v_{0}\right)}\right) / 2 v_{m}\right)^{2}},
$$

where $\alpha=d^{2} / 36 c$ and the initial porosity of granite sample in this paper is $\varphi_{0}=0.6 \%$. If the rock microstructure under different conditions has a little change, $\alpha$ is a constant. The value of $\alpha$ can be obtained with initial porosity and permeability.

Although it is commonly known that wave velocity in rock decreases with increasing temperature, there are no effective models to forecast wave velocity at different temperatures at present. This is because the changing mechanism of rock microstructure with temperature is rather complex. It is hard to establish an effective temperature-wave velocity model. But some acoustic testing technique can be used at 
TABLE 1: The basic parameters of granite specimens.

\begin{tabular}{|c|c|c|c|c|}
\hline \multirow{2}{*}{ Specimen number } & \multicolumn{2}{|c|}{ Size } & \multirow{2}{*}{ Mass (g) } & \multirow{2}{*}{ Density $\left(\mathrm{g} / \mathrm{cm}^{3}\right)$} \\
\hline & Height $(\mathrm{mm})$ & Diameter $(\mathrm{mm})$ & & \\
\hline 1 & 99.92 & 50 & 509.84 & 2.60 \\
\hline 2 & 100.25 & 50.11 & 511.40 & 2.59 \\
\hline 3 & 100.14 & 50.01 & 510.91 & 2.60 \\
\hline
\end{tabular}

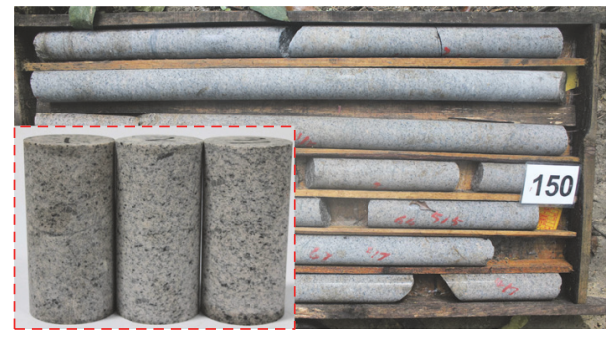

FIGURE 1: Core specimens.

high temperature; the wave velocity can be obtained through test. Therefore, there is no temperature parameter in (6).

There are many ways to change rock porosity, such as loading and heating. Benson et al. [26, 27] change the porosity via loading. In this study, heat treatment is used to change the porosity. Wave velocity and permeability will also change with porosity. The theoretical values will compare with experimental results to confirm the rationality of the model.

\section{Experiments}

3.1. Specimens. The rock used in experiment is granite from advanced exploration 36\# drill hole of Maluanshan tunnel as shown in Figure 1. The basic parameters of granite specimens are shown in Table 1.

3.2. Test Equipment. RTR-1000 rapid triaxial rock testing system of Geotechnical Consulting \& Testing Systems, LLC, shown in Figure 2, is used to measure the wave velocity and permeability. The testing system includes console, load platform, cell pressure intensifier, pore pressure intensifier, permeameter, and external source gas. $\mathrm{SX}_{2}-12-12 \mathrm{~A}$ box-type high temperature stove is used to heat specimens. The heating rate of stove is up to $10^{\circ} \mathrm{C} / \mathrm{min}$ and accuracy of steady temperature control $\leqslant 1^{\circ} \mathrm{C}$.

3.3. Test Procedures. (1) Drying of specimens: since the granite permeability measured in the experiment is very low, $\mathrm{N}_{2}$ is adopted as permeability media. Therefore, the specimens need to be dried before measuring permeability at normal temperature. Granite specimens will be dried at $50^{\circ} \mathrm{C}$ in vacuum oven. Drying time is over 12 hours until rock quality does not change. Specimens are naturally cooled to normal temperature in oven.

(2) Permeability measurement: pulse decay method is used to measure the permeability in this experiment. The dried specimens are weighed. Under conditions of axial stress
$0.5 \mathrm{MPa}$, confining pressure $5 \mathrm{MPa}, \mathrm{N}_{2}$ is injected to saturate the specimens. The pore pressure of one side of specimen remains unchanged while the other side is reduced. The permeability at this condition can be obtained from the osmotic pressure difference between two sides over treating time using

$$
k=\mu \beta V\left(\frac{\ln \left(\Delta P_{i} / \Delta P_{f}\right)}{2 \Delta t(A / L)}\right),
$$

where $\mu$ is coefficient of kinetic viscosity, $\mathrm{Pa} \cdot \mathrm{s} ; \beta$ is coefficient of volume compressibility, $\mathrm{Pa}^{-1} ; V$ is volume of pressure vessel, $\mathrm{m}^{3} ; \Delta P_{i}$ is initial osmotic pressure difference, $\mathrm{Pa} ; \Delta P_{f}$ is final osmotic pressure difference, $\mathrm{Pa} ; \Delta t$ is testing time, $\mathrm{s} ; A$ is initial sectional area of specimen, $\mathrm{m}^{2} ; L$ is initial length of specimen, $\mathrm{m}$.

As an example, the permeability of $3 \#$ specimen at $200^{\circ} \mathrm{C}$ is calculated in Figure 3. According to Brace's advice [1], the first 2 minutes of data at nonsteady seepage stage needs to be abandoned. The choice of points A and B is arbitrary. The nitrogen coefficient of kinetic viscosity is $\mu=1.78 \times$ $10^{-5} \mathrm{~Pa} \cdot \mathrm{s}$, the nitrogen coefficient of volume compressibility at this state is $\beta=2.01 \times 10^{-6} \mathrm{~Pa}^{-1}$, the pressure vessel volume is $V=5 \times 10^{-6} \mathrm{~m}^{3}$, the initial osmotic pressure difference is $\Delta P_{i}=4.072 \times 10^{5} \mathrm{~Pa}$, the final osmotic pressure difference is $\Delta P_{f}=3.967 \times 10^{5} \mathrm{~Pa}$, the testing time is $\Delta t=900 \mathrm{~s}$, the initial specimen sectional area is $A=2.501 \times 10^{-3} \mathrm{~m}^{2}$, and the initial specimen length is $L=0.10014 \mathrm{~m}$. According to (7), the permeability of $3 \#$ specimen at $200^{\circ} \mathrm{C}$ can be obtained $k=1.32 \times 10^{-19} \mathrm{~m}^{2}$.

(3) Wave velocity anisotropy measurement: $\mathrm{P}$ and $\mathrm{S}$ wave velocities of specimens are measured by ultrasonic probes inside the pressure heads of RTR-1000. Honey is used as coupling agent. Wave velocities are taken with an average of 5 measurements.

(4) High temperature processing: after wave velocity anisotropy measurement, specimens are processed by high temperature stove. Heating rate is maintained at $2^{\circ} \mathrm{C} / \mathrm{min}$. When the temperature reaches $100^{\circ} \mathrm{C}$, the specimens are kept at a uniform temperature for 3 hours and then are naturally cooled to normal temperature in stove.

(5) Repeat steps (2), (3), and (4), and the heat treatment temperature reaches $150^{\circ} \mathrm{C}, 200^{\circ} \mathrm{C}, 250^{\circ} \mathrm{C}, 300^{\circ} \mathrm{C}, 350^{\circ} \mathrm{C}$, $400^{\circ} \mathrm{C}, 450^{\circ} \mathrm{C}, 500^{\circ} \mathrm{C}, 550^{\circ} \mathrm{C}, 600^{\circ} \mathrm{C}, 650^{\circ} \mathrm{C}$, and $700^{\circ} \mathrm{C}$ successively.

The mass, permeability, and wave velocity of all specimens are measured directly after different heat treatment temperatures. 


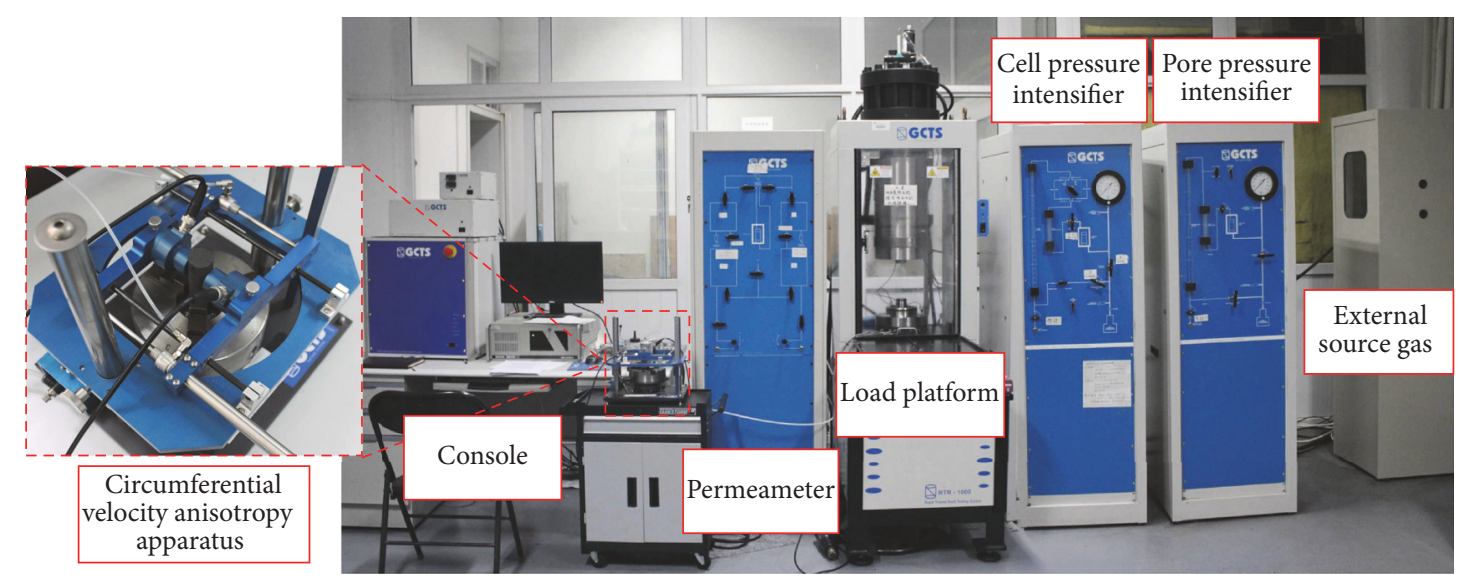

FIGURE 2: RTR-1000 rapid rock triaxial testing system.

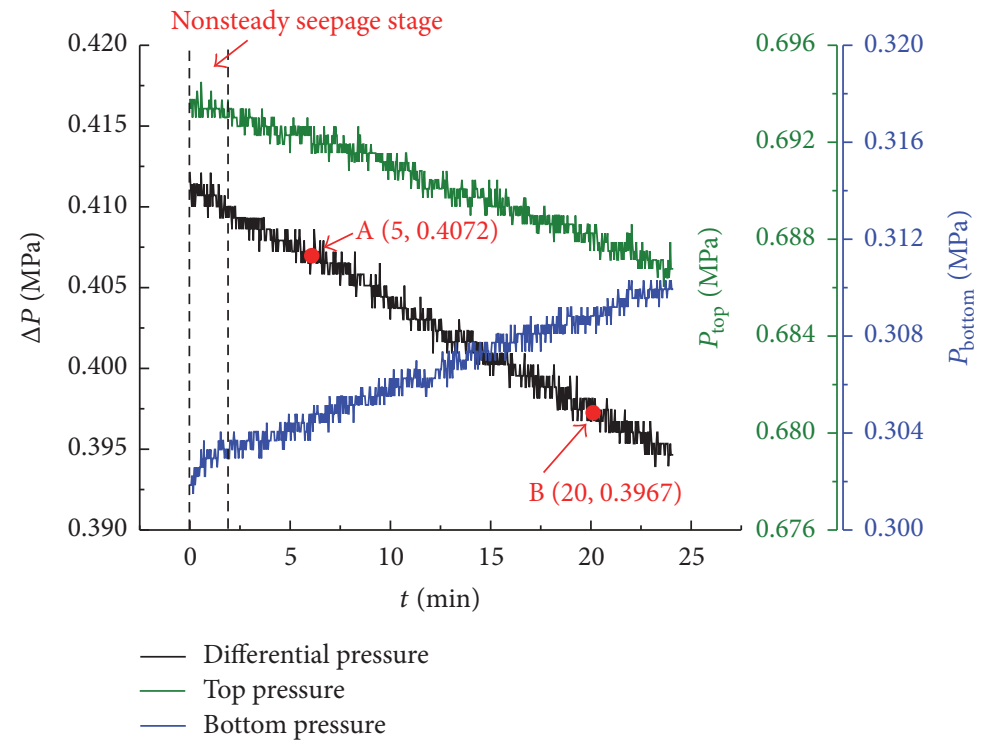

FIgURE 3: Experimental curve of pulse decay method.

\section{Experimental Results and Analysis}

4.1. Thermogravimetric Analysis. In the experimental process, the rock mass was measured after every heat treatment. It was found that, with increasing temperature, the granite mass decreases continuously, as shown in Figure 4. The change of rock mass with temperature relates to the escape of water in rock such as gravity water, bound water, and constitutional water. Figure 5 is the change curve of $1 \#$ mass and mass variable with temperature. The great mass change after $200^{\circ} \mathrm{C}, 500^{\circ} \mathrm{C}$, and $700^{\circ} \mathrm{C}$ high temperature processing corresponds to the escape of water. However, due to the low granite permeability, water cannot escape at immediate escape temperatures. Escape of water can result in the increase of rock porosity and permeability. Suppose the volume of rock is unchanged before and after heating, cumulative increment of porosity caused by water escape can be shown in Figure 6.
4.2. Change Rule of Wave Velocity with Temperature. When wave transmits in rock through the matrix and pores, its velocity in matrix is higher than the one in pores. With increasing temperature, wave velocity decreases owing to the increase in number and size of pores and hence the travel path. The change in wave velocity can be an indicator for the development cracks degree in rock. Figure 7 shows that wave velocity decreases with increasing temperature. The wave velocity drops significantly at $300^{\circ} \mathrm{C}$ and $650^{\circ} \mathrm{C}$, indicating that there are large numbers of cracks produced and developed at the two temperatures. Compared with the wave velocity at $300^{\circ} \mathrm{C}$, the one at $350^{\circ} \mathrm{C}$ showed only slight change and possible reverse trend.

4.3. Change Rule of Permeability with Temperature. The permeability increases at high temperature result from the development of pores and cracks and the increase in the rock discharge capacity, as shown in Figure 8. Permeability at 


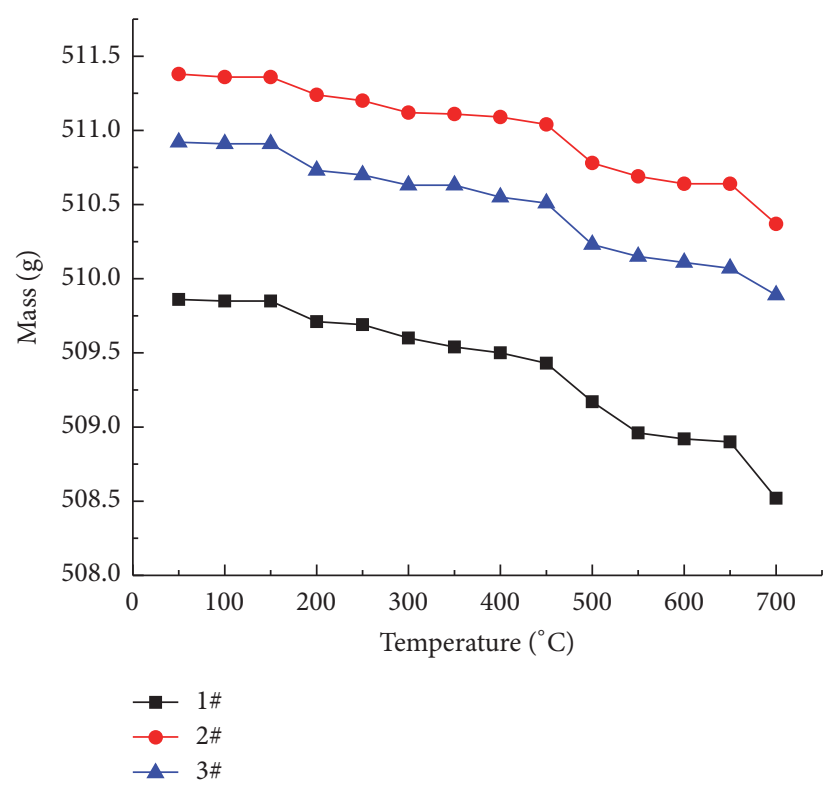

FIGURE 4: Change rule of mass with temperature.

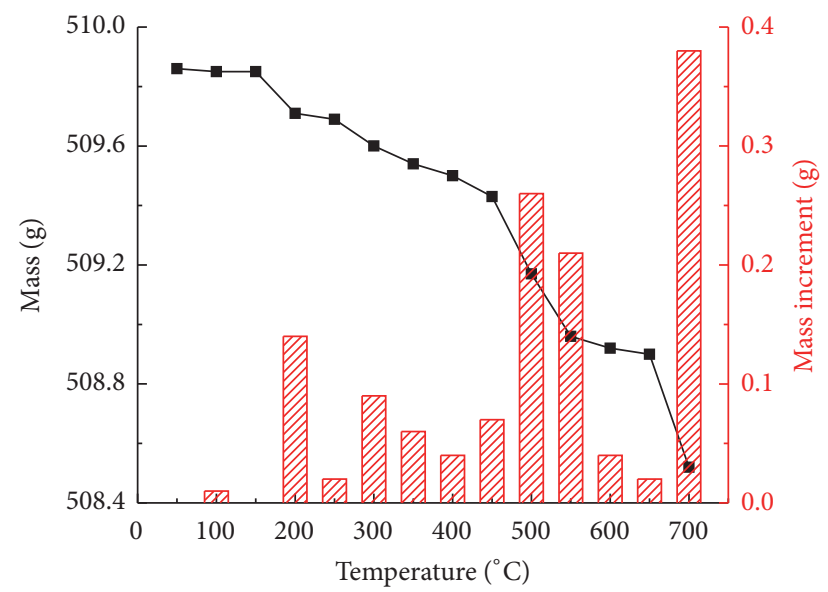

Figure 5: Change rule of $1 \#$ mass and mass variable with temperature.

$700^{\circ} \mathrm{C}$ increased by several hundred times compared to that at normal temperature.

\section{Validation of Wave \\ Velocity-Permeability Model}

According to (1), if $\alpha$ has no change, the relationship between permeability and porosity function $f(\varphi)=\varphi^{3} /(1-\varphi)^{2}$ is linear. So, porosity function $f(\varphi)$ is used as variable to analyze the relationship between porosity function $f(\varphi)$ and permeability $k$ which is shown in Figure 9.

Under close examination, the relationship between permeability and porosity function is approximatively piecewise linear function. The product of geometrical factor and particle size is various at different stages. It relates to the cracks development degree as well. As an example, the piecewise fit using $3 \#$ specimen is demonstrated. Stage 1:

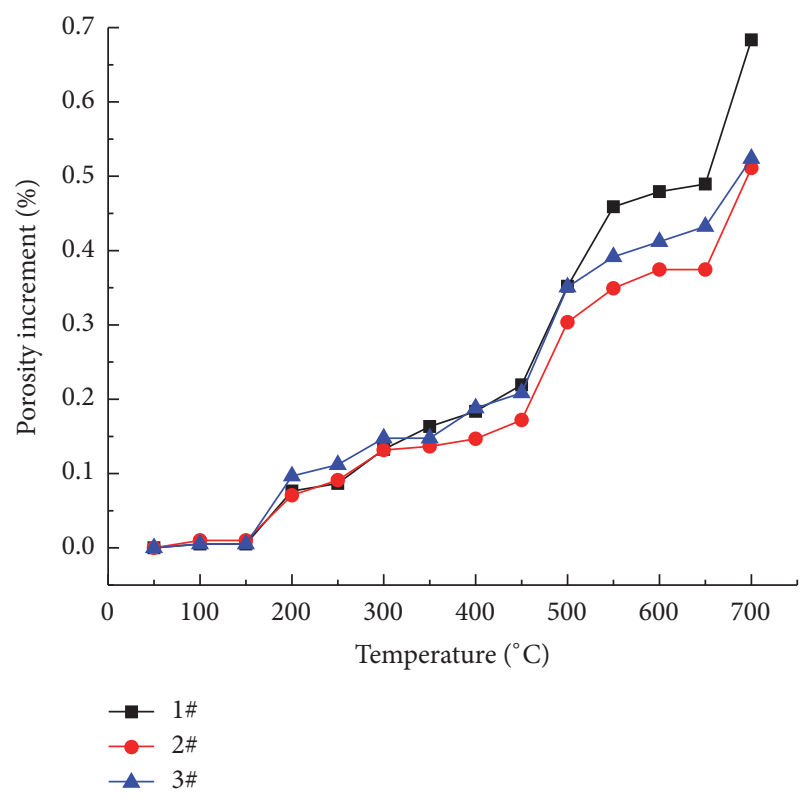

FIGURE 6: Cumulative increment of porosity caused by water escape after different heat treatment temperatures.

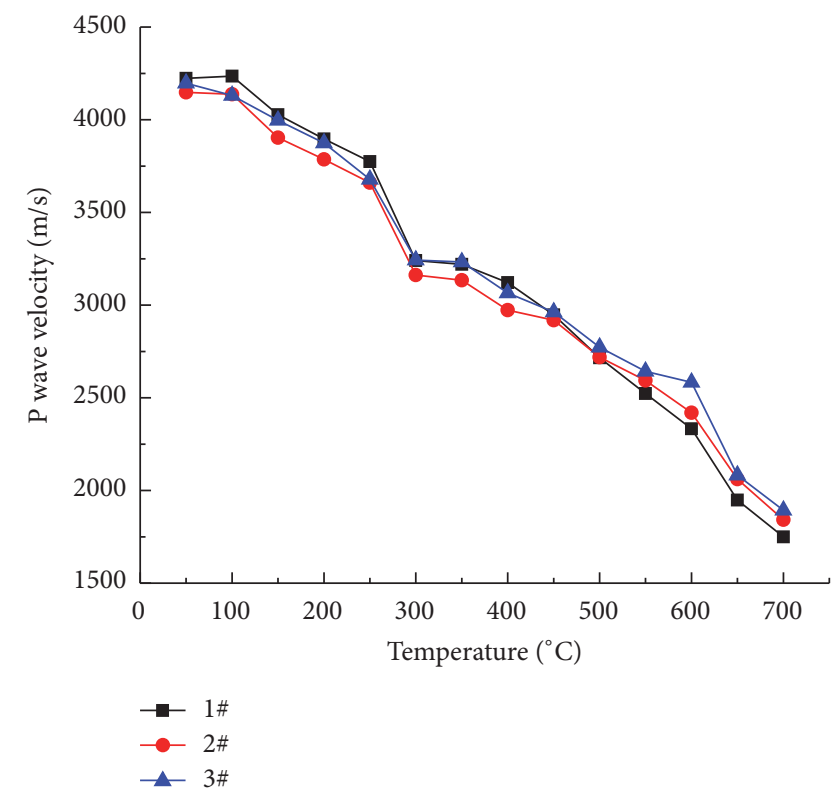

FIGURE 7: Change rule of average wave velocity with temperature.

$50^{\circ} \mathrm{C} \sim 200^{\circ} \mathrm{C}$, large slop indicates quick change in permeability with porosity function. Stage $2: 200^{\circ} \mathrm{C} \sim 400^{\circ} \mathrm{C}$, small slope shows a slow change in the permeability with porosity function. Stage 3: $400^{\circ} \mathrm{C} \sim 700^{\circ} \mathrm{C}$, slightly higher slope than that at stage 2 is obtained. Figure 10 shows that all data fit with Kozeny-Carman equation to a high degree at every stage. Table 2 shows segmentation fitting results of change rule of permeability with porosity function. Fitting degrees of all the three specimens suggest the segmentation is reasonable.

Table 2 shows that the value of $\alpha$ at stage 1 is larger than the one at stage 2 and stage 3 . It indicates that particle size is 
TABLE 2: Segmentation fitting results of change rule of permeability with $f(\varphi)$.

\begin{tabular}{|c|c|c|c|c|c|}
\hline Stage & Sample & Fitted curve & $\alpha$ & $\beta$ & $R^{2}$ \\
\hline \multirow{3}{*}{ Stage 1} & $1 \#$ & $k=13979 f(\varphi)+0.39638$ & 13979 & 0.396 & 0.881 \\
\hline & $2 \#$ & $k=14632 f(\varphi)+0.05796$ & 14632 & 0.058 & 0.940 \\
\hline & 3\# & $k=16233 f(\varphi)+0.18146$ & 16233 & 0.181 & 0.987 \\
\hline \multirow{3}{*}{ Stage 2} & $1 \#$ & $k=817 f(\varphi)+1.33911$ & 817 & 1.339 & 0.944 \\
\hline & $2 \#$ & $k=1042 f(\varphi)+1.38474$ & 1042 & 1.385 & 0.925 \\
\hline & 3\# & $k=812 f(\varphi)+1.26588$ & 812 & 1.266 & 0.998 \\
\hline \multirow{3}{*}{ Stage 3} & $1 \#$ & $k=1016 f(\varphi)+3.37391$ & 1016 & 3.374 & 0.983 \\
\hline & $2 \#$ & $k=1021 f(\varphi)+4.57822$ & 1021 & 4.578 & 0.999 \\
\hline & $3 \#$ & $k=1097 f(\varphi)+3.18941$ & 1097 & 3.189 & 0.996 \\
\hline
\end{tabular}

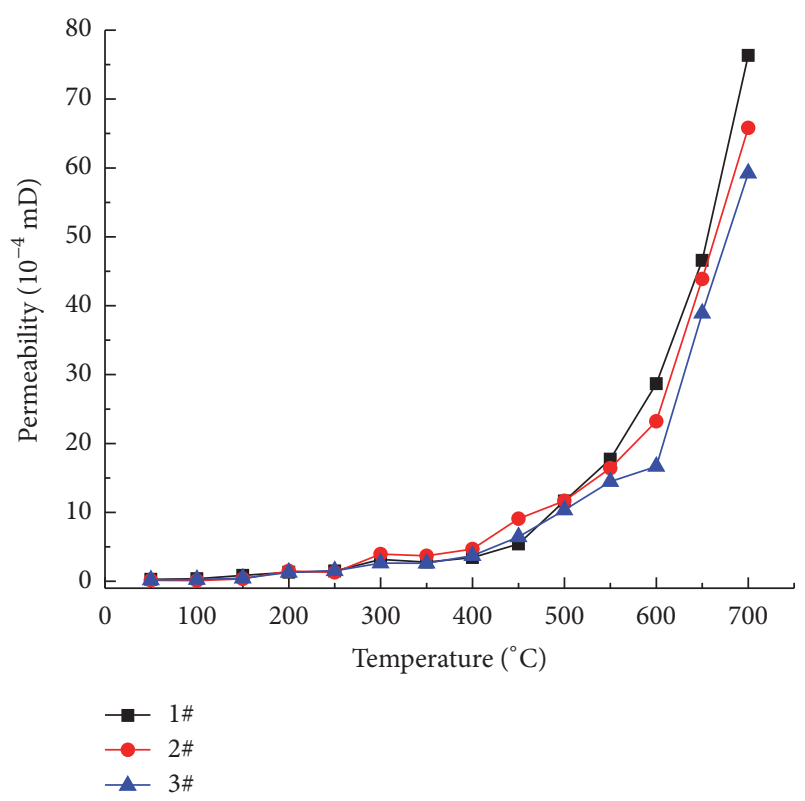

FIGURE 8: Change rule of permeability with temperature.

big at stage 1 and becomes small at stage 2 and stage 3 . Thus, this matches the real behavior. Fitting results obtained with both (1) and (6) all show intercepts that should not be from theoretical equation. The intercept should be caused by cracks development. The higher the development degree of cracks is, the bigger the intercept is. Therefore, (6) can be amended as follows:

$$
\begin{aligned}
k= & \alpha \frac{\left(\varphi_{0}-\left(m+\sqrt{m^{2}+4 v_{m}\left(v-v_{0}\right)}\right) / 2 v_{m}\right)^{3}}{\left(1-\varphi_{0}+\left(m+\sqrt{m^{2}+4 v_{m}\left(v-v_{0}\right)}\right) / 2 v_{m}\right)^{2}} \\
& +\beta
\end{aligned}
$$

where $\beta$ is a value related to crack development degree of cracks; $\alpha$ and $\beta$ need to be determined by experiments.

Substituting $\alpha$ and $\beta$ of Table 2 into (8), the fitting results are compared with experimental data in Figure 11. Given some wave velocities and the corresponding permeabilities through experiments, the other permeabilities at different temperatures and wave velocities can be obtained.

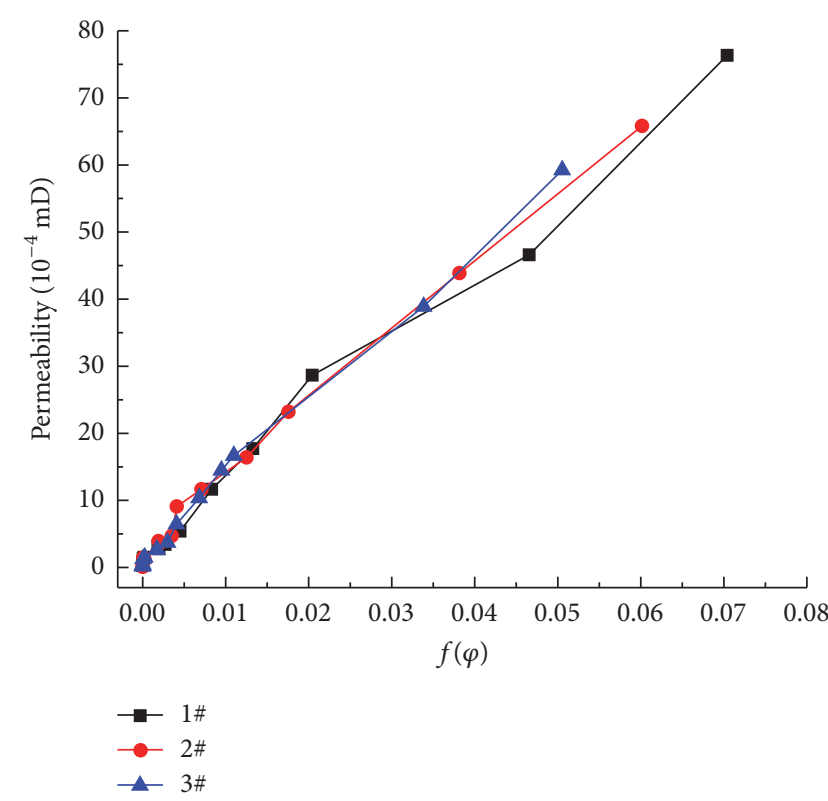

FIGURE 9: Change rule of permeability with $f(\varphi)$.

\section{Sensitivity Analysis}

A wave velocity-permeability model has been established in Section 4. In this section, the sensitivity analysis for some parameters in (8) will be carried out. Particle sizes of different rocks are much different. With sedimentary rock, for example, the range of particle size is from $10^{-5} \mathrm{~mm}$ to $10^{2} \mathrm{~mm}$. The range of sandstone particle size is mainly from $1 / 16 \mathrm{~mm}$ to $2 \mathrm{~mm}$. The sandstone with smaller particle size from $1 / 256 \mathrm{~mm}$ to $1 / 16 \mathrm{~mm}$ is called siltstone. The range of shale particle size is mainly less than $1 / 256 \mathrm{~mm}$. UddenWentworth scale is the most widely used size grading at present. Then, Krumbein [29] used $\Phi$ to express the scale as shown in Table 3. Conversion formula: $d=2^{-\Phi}$, where $d$ is particle size, mm. In sensitivity analysis, the values of $\Phi$ are $-2,0,2,4,6,8$, and 10 .

$\mathrm{P}$ wave velocities of some typical rocks are shown in Table 4 [30]. Therefore, in sensitivity analysis, the values of $v_{m}$ are $5000,6000,7000$, and $8000 \mathrm{~m} / \mathrm{s}$.

$\mathrm{P}$ wave velocities of some fluids are shown in Table 5 [31]. Therefore, in sensitivity analysis, the values of $v_{\mathrm{fl}}$ are 300,600 , 900,1200 , and $1500 \mathrm{~m} / \mathrm{s}$. 


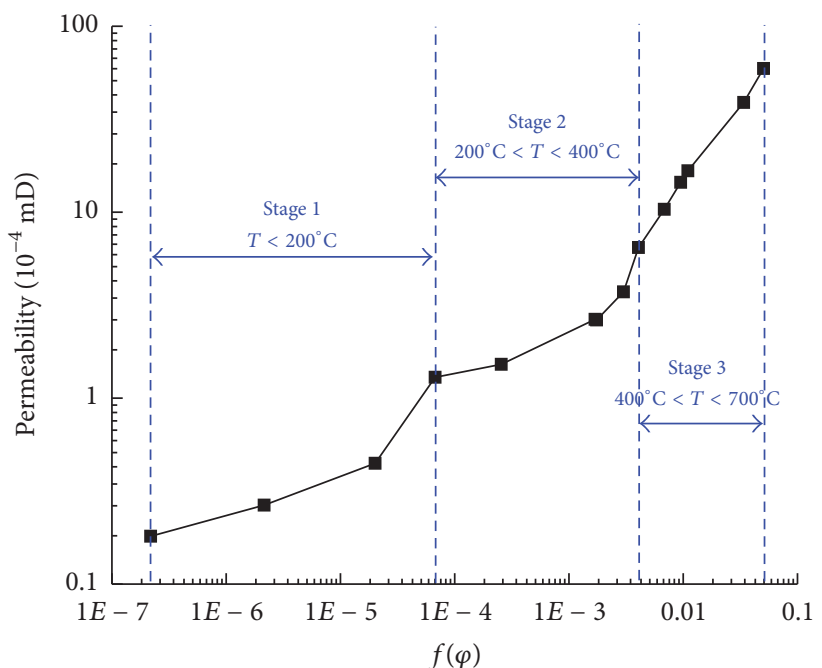

(a) 3\#

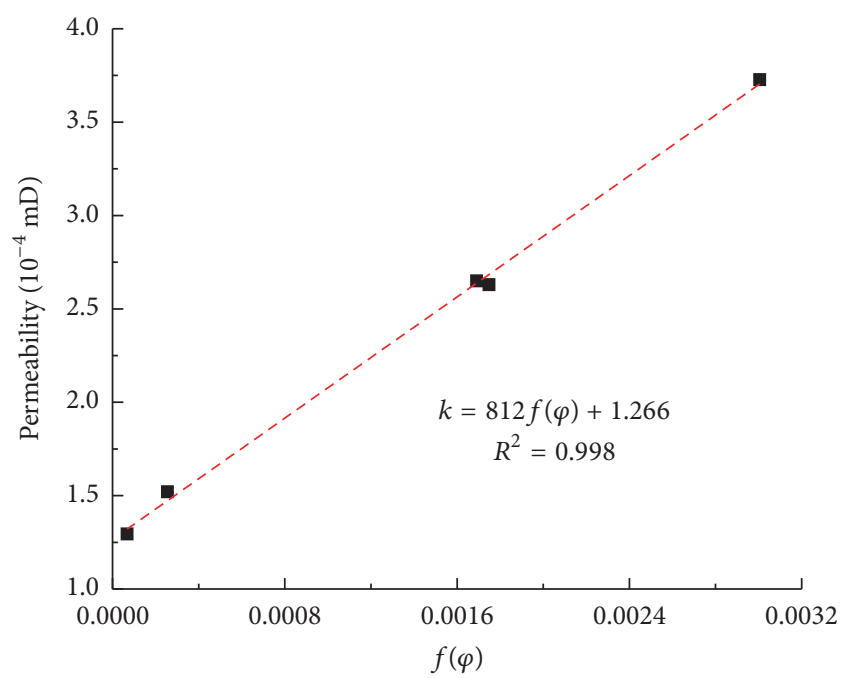

- Experimental data

- - - Fitted curve

(c) Stage 2

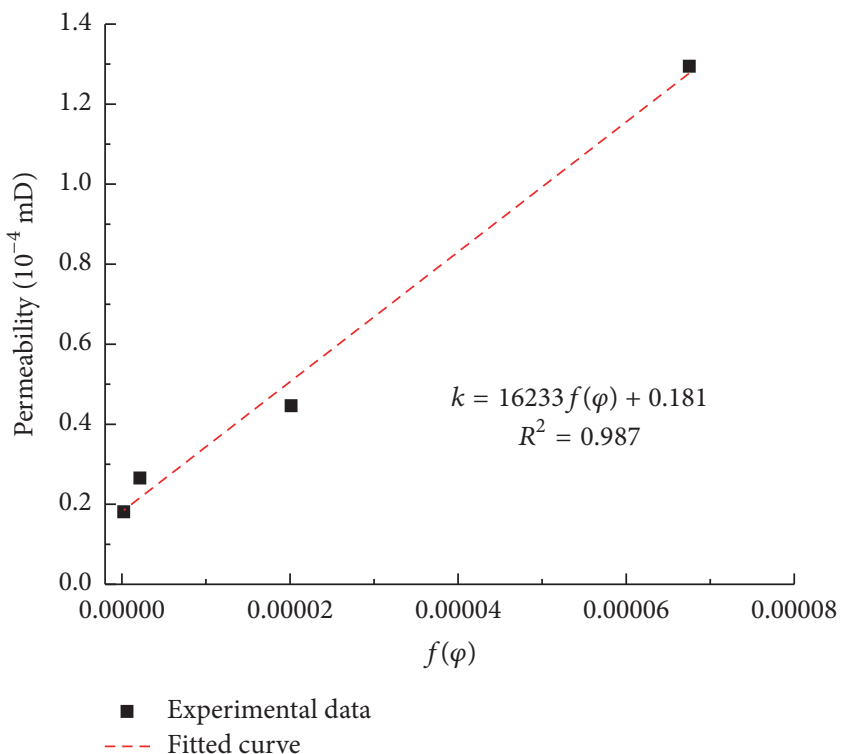

(b) Stage 1

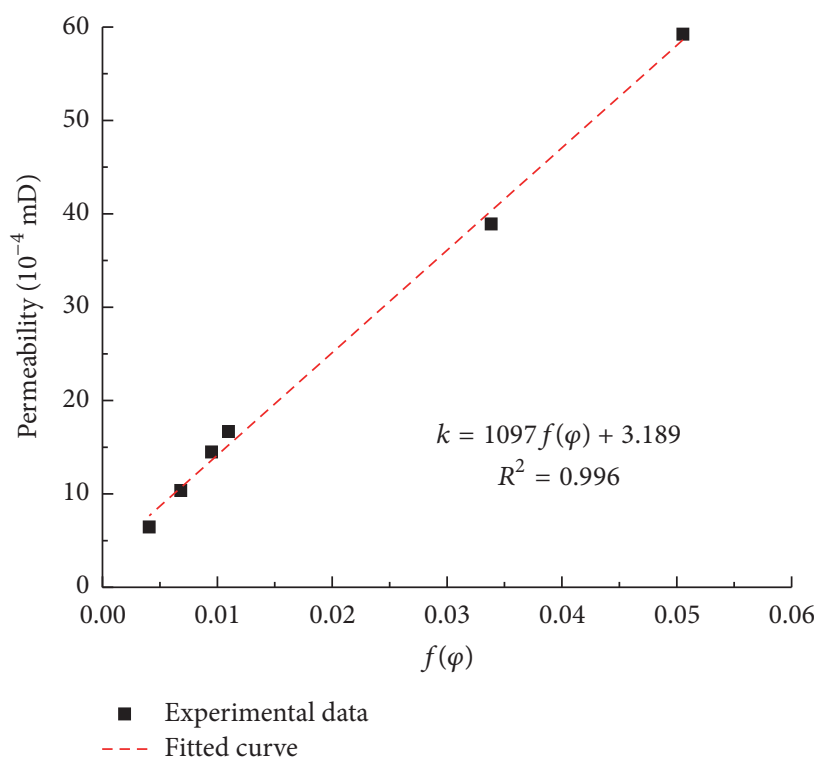

(d) Stage 3

FIGURE 10: Segmentation fitting of change rule of permeability with $f(\varphi)$ of 3\# specimen.

Figure 12 shows the effect of particle size on permeability when $v_{m}=6000 \mathrm{~m} / \mathrm{s}$ and $v_{\mathrm{fl}}=600 \mathrm{~m} / \mathrm{s}$. It clearly indicates that $\Phi$ has great effects on permeability, as shown in Figure 12. The larger $\Phi$ is, the higher the permeability will be.

Figure 13 shows the effect of $v_{m}$ on permeability when $\Phi=2$ and $v_{\mathrm{fl}}=600 \mathrm{~m} / \mathrm{s}$. The larger $v_{m}$ is, the higher the permeability will be. When $v_{r}$ is bigger, $v_{m}$ has nearly no effect on permeability. The smaller $v_{r}$ is, the greater the effect of $v_{m}$ on permeability will be.

Figure 14 shows the effect of $v_{\mathrm{fl}}$ on permeability when $\Phi=2$ and $v_{m}=6000 \mathrm{~m} / \mathrm{s}$. The larger $v_{\mathrm{fl}}$ is, the larger the permeability will be. When $v_{r}$ is larger, $v_{\mathrm{fl}}$ has nearly no effect on permeability. The smaller $v_{r}$ is, the greater the effect of $v_{\mathrm{fl}}$ on permeability will be.

\section{Conclusions}

(1) In this paper, porosity is used as medium to establish a wave velocity-permeability model. Through the new model, the permeability of rock at any temperature can be obtained with wave velocity.

(2) Heat treatment is used to change the microstructure of rock to confirm the new model. Mass, wave velocity, and permeability of granite are measured in experiments after high temperature processing: (a) with increasing temperature, the mass of granite decreases continuously. The change of rock mass with temperature was the escape of different forms of water from the rock. The water escape can increase the porosity. (b) The $\mathrm{P}$ and $\mathrm{S}$ wave velocities of granite decrease with increasing temperature. The wave velocity 


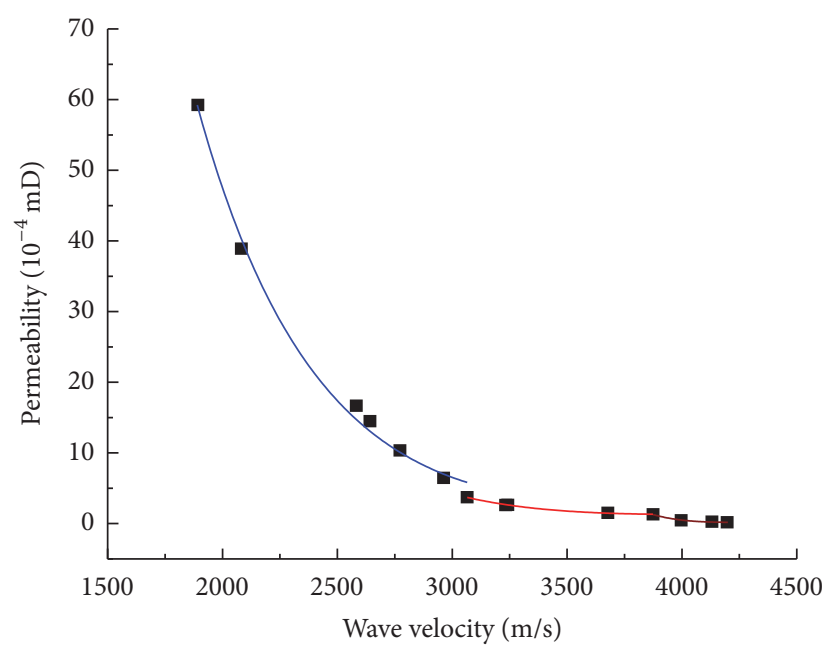

- Experimental data — Fitting curve of stage 2 Fitting curve of stage $1 \_$Fitting curve of stage 3

FIGURE 11: Segmented fitting curve of wave velocity-permeability.

TABLE 3: Udden-Wentworth scale and $\Phi$.

\begin{tabular}{lccc}
\hline$d(\mathrm{~mm})$ & $\Phi$ & $d(\mathrm{~mm})$ & $\Phi$ \\
\hline 256 & -8 & $1 / 4$ & 2 \\
128 & -7 & $1 / 8$ & 3 \\
64 & -6 & $1 / 16$ & 4 \\
32 & -5 & $1 / 32$ & 5 \\
16 & -4 & $1 / 64$ & 6 \\
8 & -3 & $1 / 128$ & 7 \\
4 & -2 & $1 / 256$ & 8 \\
2 & -1 & $1 / 512$ & 9 \\
1 & 0 & $1 / 1024$ & 10 \\
$1 / 2$ & 1 & $1 / 2048$ & 11 \\
\hline
\end{tabular}

TABLE 4: P wave velocities of some typical rocks.

\begin{tabular}{lc}
\hline Lithology & $v_{m}(\mathrm{~m} / \mathrm{s})$ \\
\hline Sandstone & $5480 \sim 5950$ \\
Limestone & $6400 \sim 7000$ \\
Dolomite & $7000 \sim 7925$ \\
\hline
\end{tabular}

TABLE 5: P wave velocity of some fluids.

\begin{tabular}{lccc}
\hline Fluid & $v_{\mathrm{fl}}(\mathrm{m} / \mathrm{s})$ & Fluid & $v_{\mathrm{fl}}(\mathrm{m} / \mathrm{s})$ \\
\hline Air & $331(273 \mathrm{~K})$ & Water & $1497(298 \mathrm{~K})$ \\
$\mathrm{CO}_{2}$ & $259(273 \mathrm{~K})$ & Drilling slurry & 1830 (mean value) \\
$\mathrm{CH}_{4}$ & $430(273 \mathrm{~K})$ & Petroleum & $1295(307 \mathrm{~K})$ \\
$\mathrm{SO}_{2}$ & $213(273 \mathrm{~K})$ & Gasoline & $1250(307 \mathrm{~K})$ \\
$\mathrm{He}$ & $965(273 \mathrm{~K})$ & Kerosene & $1420(307 \mathrm{~K})$ \\
\hline
\end{tabular}

drops significantly at $300^{\circ} \mathrm{C}$ and $650^{\circ} \mathrm{C}$ and showed only slight change at $350^{\circ} \mathrm{C}$. (c) The permeability of granite increases with increasing temperature, and the rate of the permeability increase also rises with temperature. Permeability at $700^{\circ} \mathrm{C}$

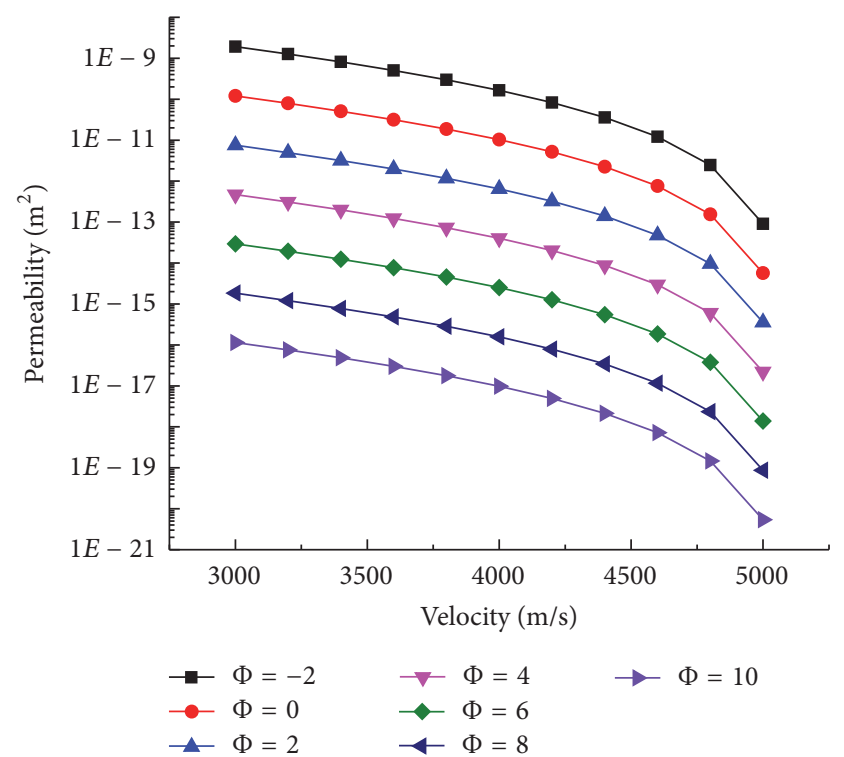

FIGURE 12: The effect of particle size on permeability.

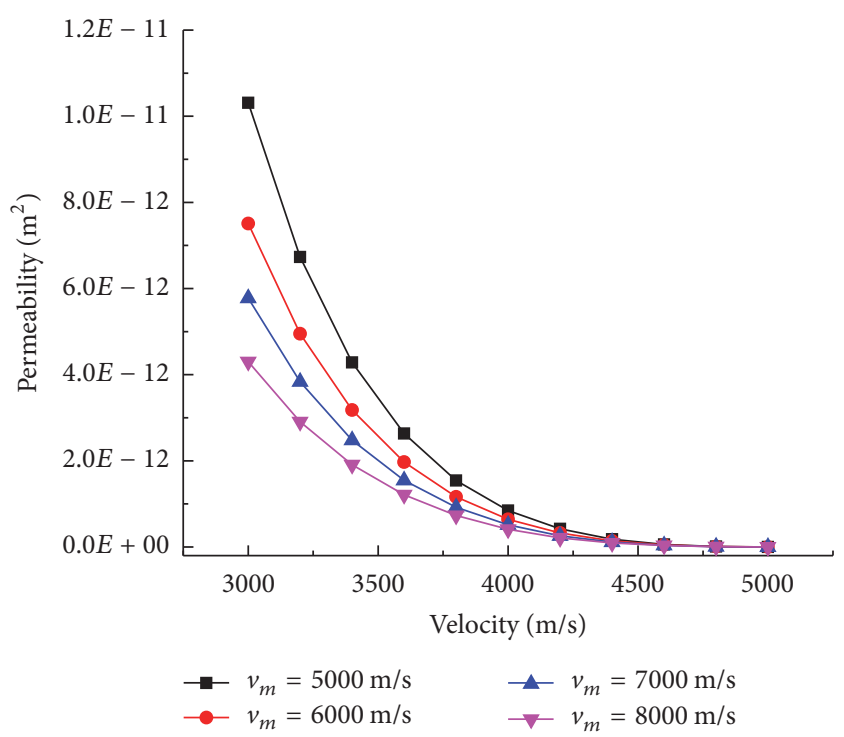

FIGURE 13: The effect of wave velocity in rock matrix on permeability.

increased by several hundred times than that at normal temperature.

(3) Using modified wave velocity-porosity empirical formula and Kozeny-Carman equation, a new wave velocitypermeability model is established. If some wave velocities and their corresponding permeabilities are known through experiments, we can obtain the other permeabilities at different temperatures and wave velocities by this model.

\section{Conflicts of Interest}

The authors declare that there are no conflicts of interest regarding the publication of this paper. 


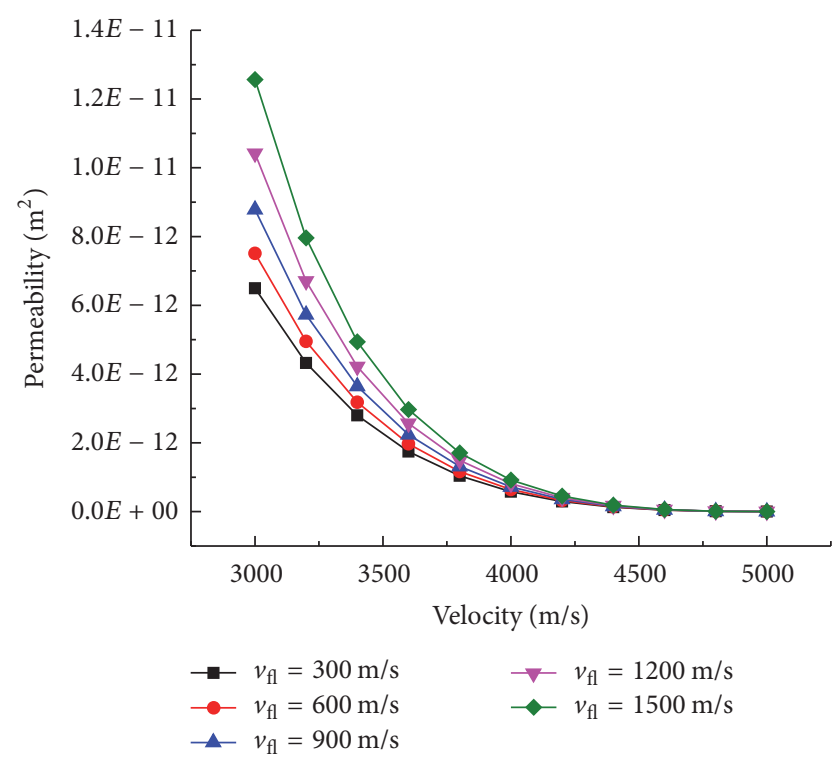

FIGURE 14: The effect of wave velocity in pore fluid on permeability.

\section{Acknowledgments}

This study was financially supported by the National Natural Science Foundation of China (51622404, 51374215, and 11572343), the State Key Research Development Program of China (2016YFC0801404), and Outstanding Young Talents of "Ten Thousand People Plan". Additionally, we would like to thank Professor Ye from California State University and Dr. Yu from Columbia University, who provided language guide for the paper.

\section{References}

[1] W. F. Brace, J. B. Walsh, and W. T. Frangos, "Permeability of granite under high pressure," Journal of Geophysical Research: Atmospheres, vol. 73, no. 6, pp. 2225-2236, 1968.

[2] R. L. Kranz, J. S. Saltzman, and J. D. Blacic, "Hydraulic diffusivity measurements on laboratory rock samples using an oscillating pore pressure method," International Journal of Rock Mechanics and Mining Sciences, vol. 27, no. 5, pp. 345-352, 1990.

[3] Y. Wang, L. I. Xiao, and H. U. Ruilin, "Review of reasearch process and application of ultrasonic testing for rock and soil," Journal of Engineering Geology, 2015.

[4] Y. Nara and K. Kaneko, "The change of P-wave velocity with temperature and humidity in granite," in Proceedings of the International Symposium of the International Society for Rock Mechanics (Eurock '06), pp. 435-441, May 2006.

[5] U. Kuila, D. N. Dewhurst, A. F. Siggins, and M. D. Raven, "Stress anisotropy and velocity anisotropy in low porosity shale," Tectonophysics, vol. 503, no. 1-2, pp. 34-44, 2011.

[6] E. W. Qiao and W. H. Zhao, "A laboratory study of seismic wave velocity in sandstone bearing differential pore fluids at different pressures," Chinese Journal of Geophysics, vol. 55, no. 12, pp. 4257-4265, 2012 (Chinese).

[7] M. R. Wyllie, A. R. Gregory, and L. W. Gardner, "Elastic wave velocities in heterogeneous and porous media," Geophysics, vol. 21, no. 1, pp. 41-70, 1956.
[8] M. R. Wyllie, A. R. Gregory, and G. H. Gardner, "An experimental investigation of factors affecting elastic wave velocities in porous media," Geophysics, vol. 23, no. 3, pp. 459-493, 1958.

[9] M. R. Wyllie, G. H. Gardner, and A. R. Gregory, "Studies of elastic wave attenuation in porous media," Geophysics, vol. 27, no. 5, pp. 569-589, 1962.

[10] L. Raymer, E. Hunt R, and S. Gardner J, "An improved sonic transit time-to-porosity transform," in Proceedings of the SPWLA 21st annual logging symposium. Society of Petrophysicists and Well-Log Analysts, 1980.

[11] P. C. Carman, "L'écoulement des gaz à travers les milieux poreux," Institut national des sciences et techniques nucléaires, 1961.

[12] O. Coussy, Acoustics of porous media, Editions Technip, 1987.

[13] J. P. Zuo, H. P. Xie, and H. W. Zhou, "SEM in situ investigation on thermal cracking behaviour of Pingdingshan sandstone at elevated temperatures," Geophysical Journal International, vol. 181, no. 2, pp. 593-603, 2010.

[14] J. P. Zuo, H. P. Xie, and W. H. Zhou, "Investigation of mesofailure behavior of rock under thermal-mechanical coupled effects based on high temperature SEM," Science China Physics, Mechanics and Astronomy, pp. 1-8, 2012.

[15] J.-P. Zuo, H.-P. Xie, F. Dai, and Y. Ju, “Three-point bending test investigation of the fracture behavior of siltstone after thermal treatment," International Journal of Rock Mechanics and Mining Sciences, vol. 70, pp. 133-143, 2014.

[16] W. Zhang, Q. Sun, S. Hao, J. Geng, and C. Lv, "Experimental study on the variation of physical and mechanical properties of rock after high temperature treatment," Applied Thermal Engineering, vol. 98, pp. 1297-1304, 2016.

[17] J. S. O. Lau, B. Gorski, and R. Jackson, "The effects of temperature and water-saturation on mechanical properties of Lac du Bonnet pink granite," in 8th ISRM Congress. International Society for Rock Mechanics, 1995.

[18] Y. Inada, N. Kinoshita, A. Ebisawa, and S. Gomi, "Strength and deformation characteristics of rocks after undergoing thermal hysteresis of high and low temperatures," International journal of rock mechanics and mining sciences \& geomechanics abstracts, vol. 34, no. 3-4, 1997.

[19] V. Rocchi, P. R. Sammonds, and C. R. J. Kilburn, "Flow and fracture maps for basaltic rock deformation at high temperatures," Journal of Volcanology and Geothermal Research, vol. 120, no. 1-2, pp. 25-42, 2003.

[20] Y.-L. Chen, S.-R. Wang, J. Ni, R. Azzam, and T. M. Fernándezsteeger, "An experimental study of the mechanical properties of granite after high temperature exposure based on mineral characteristics," Engineering Geology, vol. 220, pp. 234-242, 2017.

[21] D. Y. Xi, "Physical characteristics of mineral phase change in granite," Acta Mineralogica Sinica, vol. 03, pp. 223-227, 1994.

[22] Y. Zhang, S. Y. Zhao, and G. Wu, "Meso-structure and pattern of thermal cracking of Luhui granite," Journal of Lanzhou University of Technology, vol. 06, pp. 115-118, 2010.

[23] Z. Zhang, F. Gao, and X. L. Xu, "Acoustic emission characteristics and thermodynamic coupling model of granite under uniaxial compression," Chinese Journal of Underground Space and Engineering, vol. 01, pp. 70-74, 2010.

[24] Y. Chen, X. Wu, and F. Zhang, "Experiments on thermal fracture in rocks," Chinese Science Bulletin, vol. 44, no. 17, pp. 1610-1612, 1999 (Chinese). 
[25] Y. Zhang, Y. Zhao, Z. Wan, F. Qu, F. Dong, and Z. Feng, "Experimental study on effect of pore pressure on feldspar fine sandstone permeability under different temperatures," Yanshilixue Yu Gongcheng Xuebao/Chinese Journal of Rock Mechanics and Engineering, vol. 27, no. 1, pp. 53-58, 2008.

[26] P. Benson, A. Schubnel, S. Vinciguerra, C. Trovato, P. Meredith, and R. P. Young, "Modeling the permeability evolution of microcracked rocks from elastic wave velocity inversion at elevated isostatic pressure," Journal of Geophysical Research: Solid Earth, vol. 111, no. 4, Article ID B04202, 2006.

[27] P. M. Benson, P. G. Meredith, and A. Schubnel, "Role of void space geometry in permeability evolution in crustal rocks at elevated pressure," Journal of Geophysical Research: Solid Earth, vol. 111, no. 12, Article ID B12203, 2006.

[28] P. C. Carman, Flow of Gases through Porous Media, Butterworths Scientific Publications, London, UK, 1956.

[29] W. C. Krumbein, "Size frequency distributions of sediments," Journal of Sedimentary Research, vol. 4, no. 2, 1934.

[30] G. Mavko, T. Mukerji, and J. Dvorkin, The Rock Physics Handbook: Tools for Seismic Analysis in Porous Media, Cambridge University Press, Cambridge, UK, 2nd edition, 2009.

[31] J. H. Schön, "Rocks-Their Classification and General Properties," in Physical Properties of Rocks - Fundamentals and Principles of Petrophysics, vol. 65 of Developments in Petroleum Science, pp. 1-19, Elsevier, 2015. 

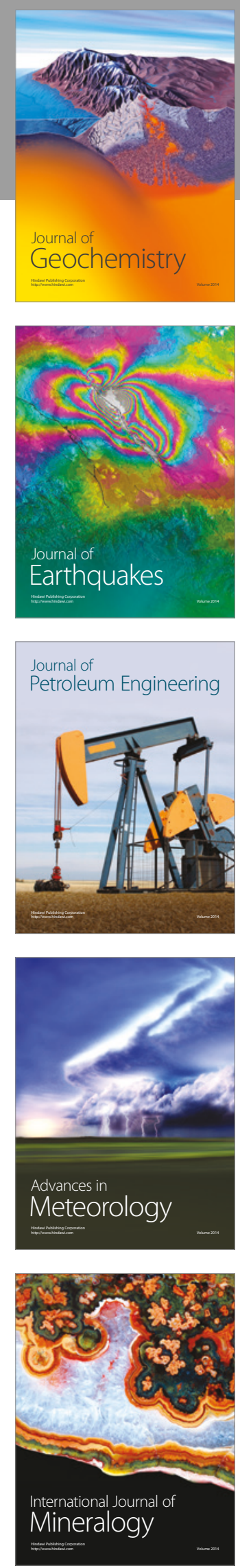
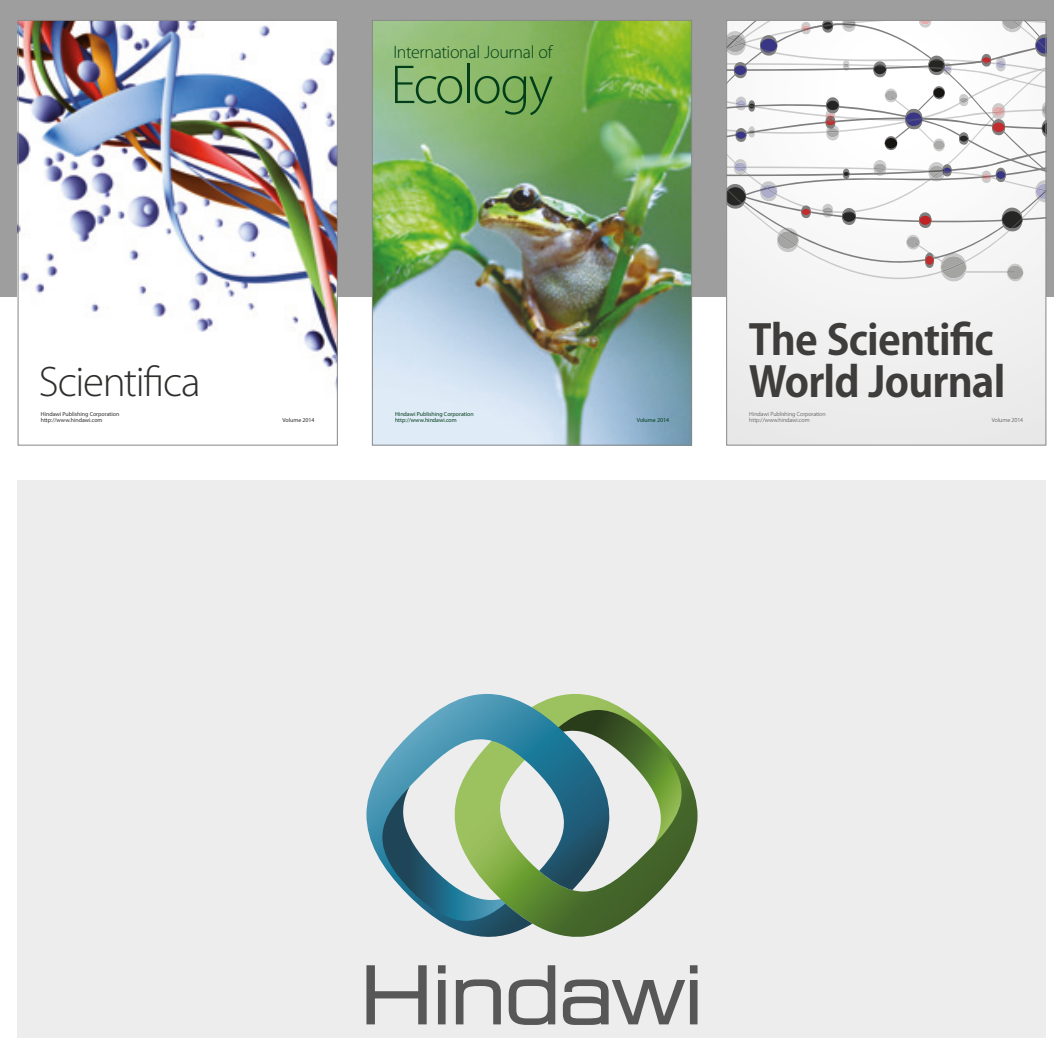

Submit your manuscripts at

https://www.hindawi.com
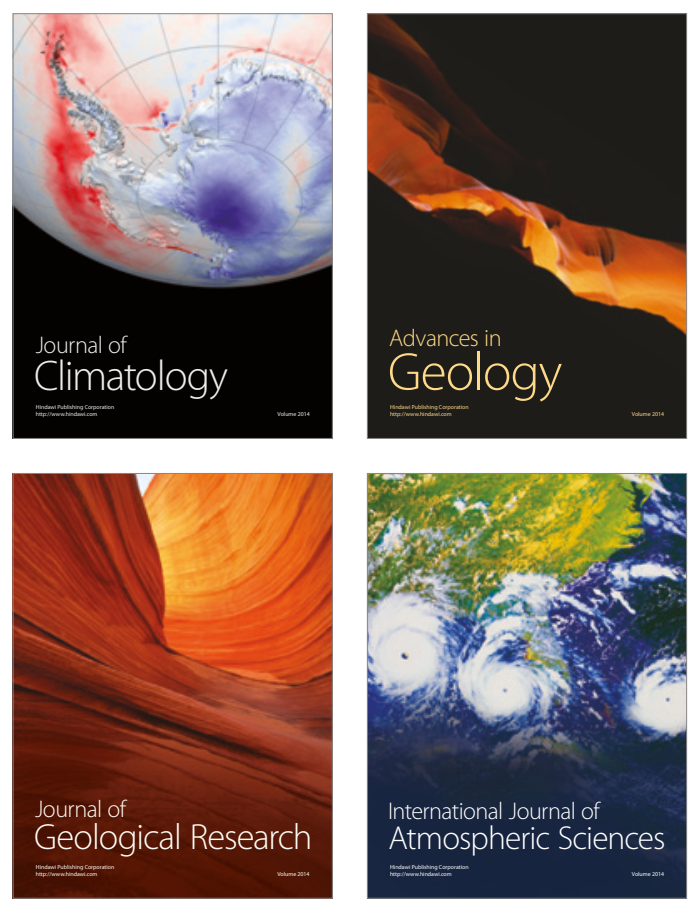

The Scientific

World Journal
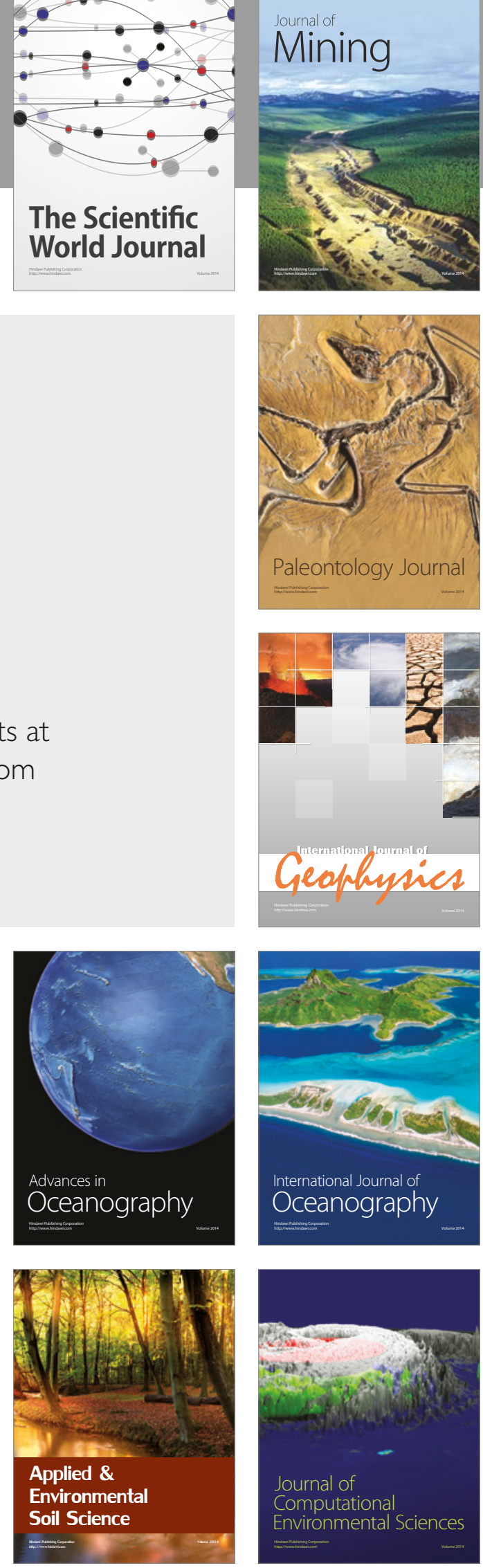